\title{
La aplicación de la canasta comercial transfronteriza como medida de reactivación comercial en Carchi - Ecuador. Un análisis de proyecciones con tendencia polinómica.
}

\section{The application of the cross-border commercial basket as a measure of commercial reactivation in Carchi - Ecuador. An analysis of projections with a polynomial tendency.}

Jean Pierre Rosero López

Universidad Internacional del Ecuador, Ecuador

Rodrigo Fernando Hallo Alvear

Universidad Internacional del Ecuador, Ecuador

Autor para correspondencia: jeroserolo@uide.edu.ec,rohalloal@uide.edu.ec

Fecha de recepción: 23 de julio de 2018 - Fecha de aceptación: 20 septiembre de 2018

Resumen: La devaluación del peso colombiano frente al dólar, la aplicación de salvaguardas por parte del Gobierno ecuatoriano, la baja del precio del petróleo a nivel mundial, entre otros factores, deprimió el comercio fronterizo en Ecuador, esencialmente en la provincia del Carchi donde el principal ingreso económico es por actividades comerciales con el departamento de Nariño, al sur de Colombia. Una de las medidas que adoptó el Gobierno de Rafael Correa fue la de declarar a la Provincia del Carchi como "zona deprimida" el 18 de agosto de 2015, y cuyo objetivo era que los comerciantes registrados en el Servicio de Rentas Internas SRI, puedan adquirir productos en Colombia para venderlos en su localidad a precios competitivos generando reactivación comercial. Esta investigación utiliza el método analítico y sistémico, con un enfoque técnico aplicado en dos casos de empresas del sector comercial, utilizando proyecciones con tendencia polinómica para alcanzar conclusiones diagnósticas.

Palabras clave: canasta comercial transfronteriza; zona deprimida; Carchi; comercio fronterizo

\begin{abstract}
The devaluation of the Colombian peso against the dollar, the application of safeguards by the Ecuadorian government, the drop in the price of oil worldwide, among other factors, depressed border trade in Ecuador, essentially in the province of Carchi where the main income economic is by commercial activities with the department of Nariño, south of Colombia. One of the measures adopted by the Government of Rafael Correa was to declare the Province of Carchi as "depressed area" on August 18th, 2015, and whose objective was that merchants registered in the Internal Revenue Service SRI, can acquire products in Colombia to sell them in your locality at competitive prices generating commercial reactivation. This research uses the analytical and systemic method, with a technical approach applied in two cases of companies in the commercial sector, using projections with a polynomial tendency to reach diagnostic conclusions.
\end{abstract}

Key Words: cross-border commercial basket; depressed zone; Carchi, border trade 


\section{Introducción}

La devaluación del peso colombiano en más de 50\% en el año 2014 frente al dólar, la implementación de las salvaguardias por parte del gobierno central para regular el nivel general de las importaciones y equilibrar la balanza comercial, la apreciación de dólar a nivel mundial, entre otras, fueron algunas de las causas que conllevaron a una depresión en el comercio fronterizo del país, sobre todo en la provincia del Carchi al norte del Ecuador, en donde el principal ingreso económico se debe a las actividades comerciales entre esta provincia y el departamento de Nariño, al sur de Colombia.

La provincia del Carchi tiene un número reducido de industrias, de acuerdo a datos de la cartera de gobierno (Ministerio de Industrias y Productividad 2017), lo que provoca a sus ciudadanos volcarse por la actividad comercial y agrícola, siendo su principal mercado el departamento de Nariño; sin embargo, este comercio se ha visto disminuido y sus habitantes perjudicados por la baja de ingresos.

Debido a esto, una de las medidas que adoptó el gobierno de Rafael Correa fue la declaración a la provincia del Carchi en agosto de 2015 como "zona deprimida", con lo que se buscaba ayudar a los comerciantes que fueron afectados comercialmente en la zona; el Consejo Sectorial de la Producción declaró como zonas deprimidas al cantón Tulcán de la provincia del Carchi, varios cantones de la provincia de Sucumbíos, a la provincia de Orellana, a la provincia de Loja y al cantón Huaquillas de la provincia de El Oro y creó la "Canasta Comercial Transfronteriza", medida cuyo objetivo fue que los comerciantes de las zonas deprimidas puedan adquirir productos en los países vecinos (Colombia y Perú) para venderlos en su localidad a precios competitivos, generando de esta manera una reactivación de la actividad comercial. (EKOS, 2016)

Para la aplicación de esta medida se crearon cuotas de importación de acuerdo a tres segmentos en los que estaban clasificados a los comerciantes, los segmentos se establecieron con base en las ventas anuales registradas en el Servicio de Rentas Internas (SRI) durante el año 2014, con fecha de corte hasta la adopción de las distintas resoluciones. Los segmentos fueron los siguientes: Segmento 1: desde USD 0 hasta USD 20.000; Segmento 2: superior a USD 20.000 y hasta USD 160.000; y, Segmento 3: superior a USD 160.000. (COMEX, 2015)

Según (Colegio de economistas, 2017), esta medida estuvo en vigencia entre octubre del 2015 hasta mayo del 2017, y permitía a comerciantes importar productos de distinto tipo como celulares, televisores, línea blanca, entre otros, sin salvaguardias ni aranceles desde Colombia y venderlos en el Ecuador, pagando únicamente el Impuesto al Valor Agregado IVA, el Impuesto a Consumos Especiales ICE y el Fondo de Desarrollo para la Infancia FODINFA. (Importsol, 2017)

Con base en declaraciones de comerciantes de la zona y con los resultados presentados del (Ministerio de Industrias y Productividad 2017), esta medida, en primera instancia, fue dirigida de manera ambigua para los comerciantes, creando una confusión en los productos a los que iban dirigidos de acuerdo a la actividad comercial declarada en el Registro Único del Contribuyente RUC de cada comerciante; en otras palabras, y de acuerdo con datos levantados 
en campo, habían comerciantes que su RUC estaba enfocado a la comercialización de prendas de vestir y aun así tenían capacidad de realizar legalizaciones de productos de tipo tecnológico.

Según el Servicio Nacional de Aduanas del Ecuador (SENAE), 399 de los 4.770 comerciantes autorizados por el Comité de Comercio Exterior (COMEX) usaron este mecanismo para traer desde Colombia mercadería libre de impuestos y salvaguardias. (El Comercio, 2016)

\section{Métodos}

El estudio fue realizado en dos empresas de la ciudad de Tulcán, provincia de El Carchi, mismas que por cuestiones de confidencialidad de la información solicitaron mantener ocultos sus nombres, las cuales tienen la particularidad de ser de las pocas empresas que accedieron al cupo más alto de la canasta comercial transfronteriza, debido a sus declaraciones de impuesto a la renta que sobresalía de los demás beneficiarios de la canasta comercial; es decir se utilizó un muestreo no probabilístico por conveniencia.

De esta manera se tiene dos objetos de estudio, con los cuales se tomó en cuenta el resultado anual de sus ventas locales con los datos reales que fueron proporcionados, realizando en el primer escenario la proyección hacia el año 2018, teniendo la data desde el año 2011 hasta el año 2016.

El análisis de tendencia lineal es una técnica estadística utilizada para estudiar la relación entre variables. Se adapta a una amplia variedad de situaciones. En la investigación social, el análisis de proyecciones se utiliza para predecir un amplio rango de fenómenos, desde medidas económicas hasta diferentes aspectos del comportamiento humano.

Según (Render, Stair, \& Hanna, 2006), un método muy interesante para pronosticar series de tiempo con tendencia se conoce como proyección de tendencia. Esta técnica ajusta una línea de tendencia a una serie de puntos de datos históricos y entonces proyecta la línea hacia el futuro para pronósticos de mediano y largo plazo. Existen varias ecuaciones matemáticas de tendencias que pueden desarrollarse (por ejemplo, exponenciales y cuadráticas), pero en esta investigación tendencias lineales (líneas rectas). Una línea de tendencia simplemente es una ecuación de regresión lineal en la cual la variable independiente $(\mathrm{X})$ es el periodo considerado. Su modelo es:

$$
Y=b_{o}+b_{1} X
$$

En donde:

$$
\begin{gathered}
Y=\text { Valor pronosticado } \\
b_{o}=\text { Ordenada al origen } \\
b_{1}=\text { Pendiente de la línea } \\
X=\text { Periodo }(\text { por ejemplo, } X=1,2,3, \ldots, n)
\end{gathered}
$$

El criterio inicial para escoger el tipo de línea de tendencia radica en la forma que adoptan los puntos de la serie de datos. Por ejemplo, si se aprecia que la serie de datos muestra un crecimiento a tasas constantes, es probable que una tendencia lineal se ajuste mejor. En 
cambio, si el crecimiento mostrado es a tasas crecientes y decrecientes, es probable que se logran mejores ajustes con una línea de tendencia "polinómica".

La línea de tendencia polinómica asume la forma $a_{0}+a_{1 x}+a_{2 x}{ }^{2}$, donde se buscará determinar los valores de $a_{0}, a_{1 x}, a_{2 x}{ }^{2}$, que minimicen la suma de los cuadrados de las desviaciones. (Dapena Fernandez, 2015)

\section{Resultados y Discusión}

El estudio fue realizado en dos empresas de la ciudad de Tulcán bajo las características expresadas en el apartado anterior. De esta manera se tienen dos objetos de estudio, con los cuales se tomó en cuenta el resultado anual de sus ventas locales con los datos reales que fueron proporcionados, realizando en el primer escenario la proyección hacia el año 2018 con data desde el año 2011 hasta el año 2016.

En la Tabla 1 se presenta el crecimiento de la empresa 1 desde el año 2011 hasta el 2016, y aplicada la proyección se presenta los resultados esperados en el 2018.

Tabla 1: Datos de ventas de la empresa 1 periodo 2011 - 2016 y su proyección al 2018

\begin{tabular}{ccc}
\hline Año & Ventas en USD. & Crecimiento / Decrecimiento ventas \% \\
\hline 2011 & $\$ 951.915,97$ & \\
2012 & $\$ 970.954,29$ & $2,0 \%$ \\
2013 & $\$ 1.009 .792,46$ & $4,0 \%$ \\
2014 & $\$ 1.029 .988,31$ & $2,0 \%$ \\
2015 & $\$ 911.494,08$ & $-11,5 \%$ \\
2016 & $\$ 821.438,00$ & $-9,9 \%$ \\
\hline 2018 & $\$ 672.405,00$ & $-18,1 \%$
\end{tabular}

Nota: Registros de ventas obtenidos de la Empresa 1 en estudio y su proyección utilizando proyección con tendencia polinómica.

Con lo presentado en la tabla anterior, se puede evidenciar que, durante los últimos dos años de ventas reales, la empresa 1 experimentó un decrecimiento de ventas pese a la aplicación de la medida gubernamental. Es importante mencionar que el año 2017, si bien está ya cerrado, aun no se posee cifras reales y oficiales que permitan incorporar en la proyección. Además, es importante mencionar que, usando este tipo de proyección, se obtiene una cifra inferior a la presentada en el 2016.

En la Figura 1, se presenta de manera gráfica el histórico de las ventas y su respectiva proyección en la empresa 1 utilizando tendencia polinómica. 


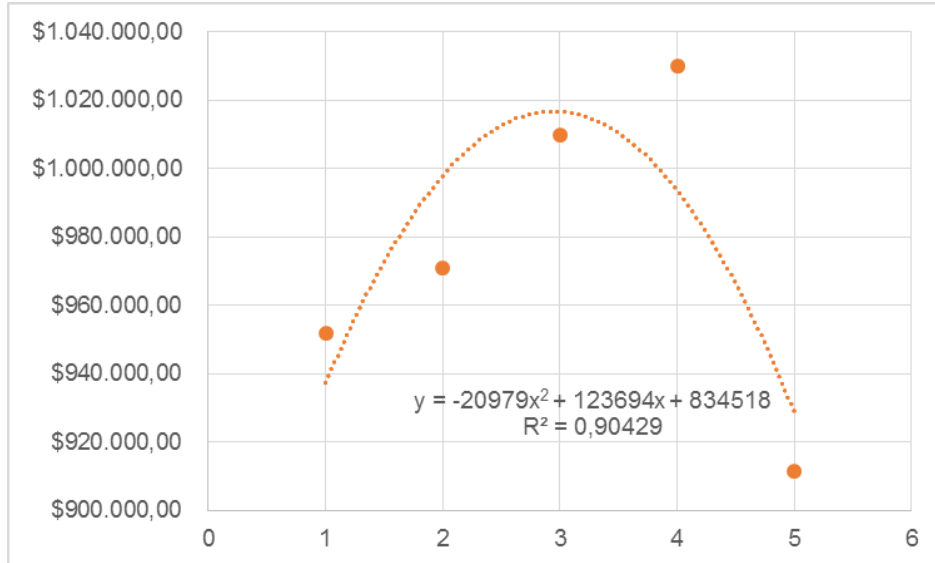

Figura 1: Cifras de ventas de la empresa 1 y su proyección utilizando tendencia polinómica Nota: Se utilizaron las cifras proporcionadas por la empresa y se aplicó la proyección con tendencia polinómica para la empresa 1.

Como se puede evidenciar, la empresa 1 empieza a decrecer en sus ventas a partir del año 2015, siendo tan pronunciado el decrecimiento que alcanzó un 11,5\% con respecto al año 2014; esto se puede atribuir a diversos factores, uno de ellos se da por la devaluación del peso colombiano casi en 50\% explicado previamente, lo cual se convirtió en un factor crítico para esta empresa debido a que el mercado principal al cual se dirige, en un $65 \%$ de su proporción de ventas era el colombiano.

Sin embargo, tal como lo expresa el gerente de la empresa 1 a quien se le realizó una entrevista corta durante la presente investigación, en la frontera norte del Ecuador siempre se ha convivido con la fluctuación cambiaria dólar-peso, lo cual incentivaba a los comerciantes de esta zona a generar una mejor oferta comercial disminuyendo sus utilidades para no perder mercado.

Pero la medida que puede considerarse de mayor afectación por los entendidos fue la aplicación de salvaguardas, debido a que los productos que comercializa esta empresa no son fabricados en el país por tanto el sobreprecio que debieron aplicar en los nuevos productos que llegaban y no estaban en inventario con precios anteriores, se debían vender con un precio más alto, lo cual generó que los clientes colombianos huyan por lo precios altos y decidan comprar en su país, debido también al factor que este país cuenta con varios Tratados de Libre Comercio y conseguían estos productos a muy bajos precios en especial con Estados Unidos.

Por otro lado, y aplicando la misma metodología, se realizó el análisis en la empresa 2 en el cual se obtuvo como resultado cifras interesantes presentadas a continuación.

Para la realización de la proyección hacia el año 2018 se tomaron de igual manera que con la empresa 1, los datos proporcionados por la organización; sus resultados son visibles en la Tabla 2 y Figura 2 donde su $R^{2}$ es de 0,904 , lo cual indica que el valor resultante es confiable debido a que este se está acercando a 1 .

Tabla 2: Datos de ventas de la empresa 2 periodo 2011 - 2016 y su proyección al 2018

\begin{tabular}{ccc}
\hline Año & Ventas en USD. & Crecimiento / Decrecimiento ventas \% \\
\hline 2011 & $\$ 808.026,70$ \\
\hline
\end{tabular}




\begin{tabular}{lll}
\hline 2012 & $\$ 832.267,50$ & $3,00 \%$ \\
2013 & $\$ 905.090,91$ & $8,75 \%$ \\
2014 & $\$ 906.901,09$ & $0,20 \%$ \\
2015 & $\$ 842.567,41$ & $-7,09 \%$ \\
2016 & $\$ 778.001,00$ & $-7,66 \%$ \\
\hline 2018 & $\$ 668.290,00$ & $-14,10 \%$ \\
\hline
\end{tabular}

Nota: Registros de ventas obtenidos de la Empresa 2 en estudio y su proyección utilizando proyección con tendencia polinómica.

Respecto a la Tabla 2 de la empresa 2, se puede evidenciar que también presenta decrecimiento a partir del año 2015; sin embargo, esta empresa tiene la particularidad de que en el año 2014 su crecimiento en ventas con respecto al año anterior se frenó drásticamente ya que en este año solo creció su nivel de ventas en 0,2\%. Posterior a la aplicación de la medida gubernamental, no se evidencia crecimiento de ventas.

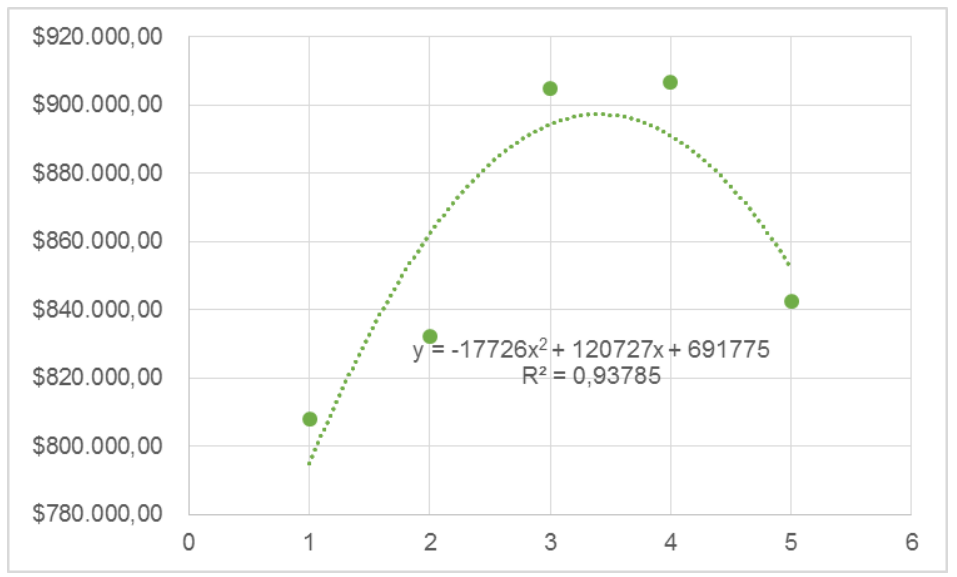

Figura 2: Cifras de ventas de la empresa 2 y su proyección utilizando tendencia polinómica Nota: Se utilizaron las cifras proporcionadas por la empresa y se aplicó la proyección con tendencia polinómica para la empresa 2.

Para la realización de la proyección hacia el año 2018 con los datos proporcionados, se puede observar en la Figura 2 que su $R^{2}$ es de 0,93, lo cual indica que el valor resultante es confiable debido a que este se está acercando a 1.

De esta forma se puede evidenciar que la empresa 2 presenta un decrecimiento promedio de $-2,8 \%$ dentro del periodo analizado. De igual forma, apalancado de la entrevista al gerente de la empresa 2, se puede evidenciar que unos de los factores influyentes para esto es la aplicación de salvaguardas, ya que los productos que comercializan son en un $97 \%$ importados generando el aumento de sus precios; sin embargo, una de las ventajas que la empresa tenía fue el suficiente stock de mercadería en comparación de su principal competencia, lo que permitió mantener precios anteriores por más tiempo.

Posterior a la presentación de estos resultados, el siguiente paso fue evaluarlos con los montos de las ventas esperadas para cada año en cada empresa, esto tomando en cuenta los años en los que aún no se aplicaba por parte del gobierno las salvaguardas ni se considera a la ciudad 
de Tulcán como "zona deprimida"; es decir, cuando el comercio se daba de manera normal. Para esto se ha realizado las proyecciones basadas en tendencias polinómicas desde las ventas anuales correspondientes al año 2011 hasta el año 2016 con base en los datos que proporcionaron, tanto la empresa 1 como la empresa 2.

En la empresa 1 se puede observar que el crecimiento de ventas con respecto al año subsiguiente fluye con promedio de crecimiento de 2,73\% anual, lo cual es un crecimiento considerable y estable considerando los montos que ingresa a la empresa. Como se puede evidenciar en la siguiente figura, la proyección de tendencia polinómica tiene una confiabilidad de $R^{2}=0,98$, es decir del $98 \%$.

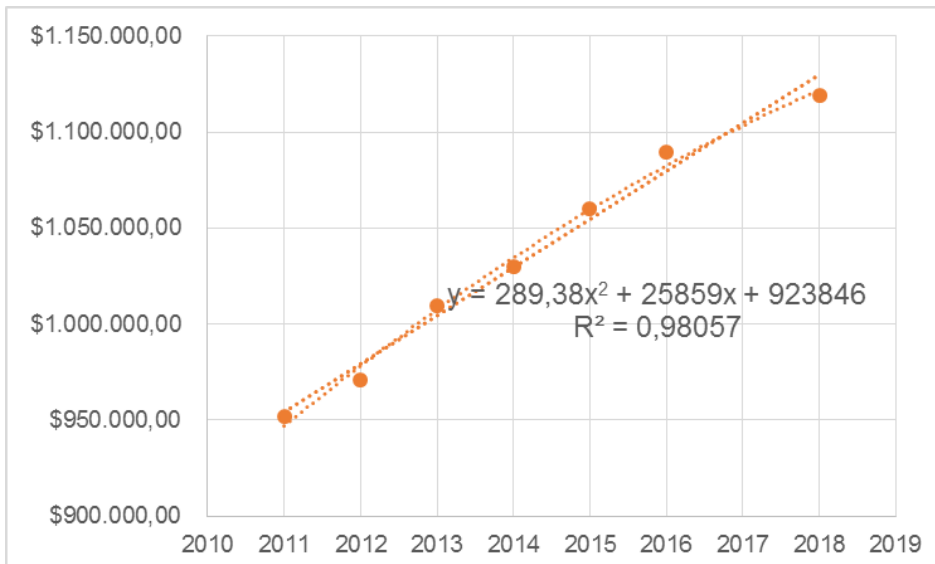

Figura 3: Comportamiento de ventas con proyección de los años 2015, 2016, 2018 de la empresa 1

Nota: Se utilizaron las cifras proporcionadas por la empresa y se aplicó la proyección con tendencia polinómica para la empresa 1.

Como se evidencia, si en el Ecuador no se hubiese aplicado medidas para la represión del comercio, las ciudades fronterizas hubiesen seguido un crecimiento normal como se lo había venido haciendo en años anteriores, la empresa 1 hubiese tenido un crecimiento no muy grande en cifras porcentuales, pero si constante.

Tabla 3: Proyección de ventas de la empresa 2 para los años 2015, 2016, 2018.

\begin{tabular}{ccc}
\hline Años & Ventas en USD. & Crecimiento / Decrecimiento ventas \% \\
2011 & $\$ 951.915,97$ & \\
2012 & $\$ 970.954,29$ & $2,0 \%$ \\
2013 & $\$ 1.009 .792,46$ & $4,0 \%$ \\
2014 & $\$ 1.029 .988,31$ & $2,0 \%$ \\
2015 & $\$ 1.060 .375,50$ & $3,0 \%$ \\
2016 & $\$ 1.089 .417,68$ & $2,7 \%$ \\
2018 & $\$ 1.119 .038,62$ & $2,7 \%$
\end{tabular}

Nota: Registros de ventas obtenidos de la Empresa 2 en estudio y su proyección utilizando proyección con tendencia polinómica. 
Mientras tanto que, para la empresa 2, los resultados hubiesen sido mejores si no se hubiese aplicaban restricciones como las salvaguardas. Mediante la proyección de tendencia polinómica se llegó a obtener una confiabilidad de $R^{2}=0,9067$, es decir del 90,67\%.

El crecimiento en ventas promedio de esta empresa es de 6,82\% durante el periodo de los años 2011 hasta el 2016 y su estimación 2018, lo cual hubiese generado mejor rentabilidad para el negocio y en el fomento del empleo, por ejemplo.

En la siguiente figura se puede evidenciar la tendencia de crecimiento proyectada de la empresa 2.

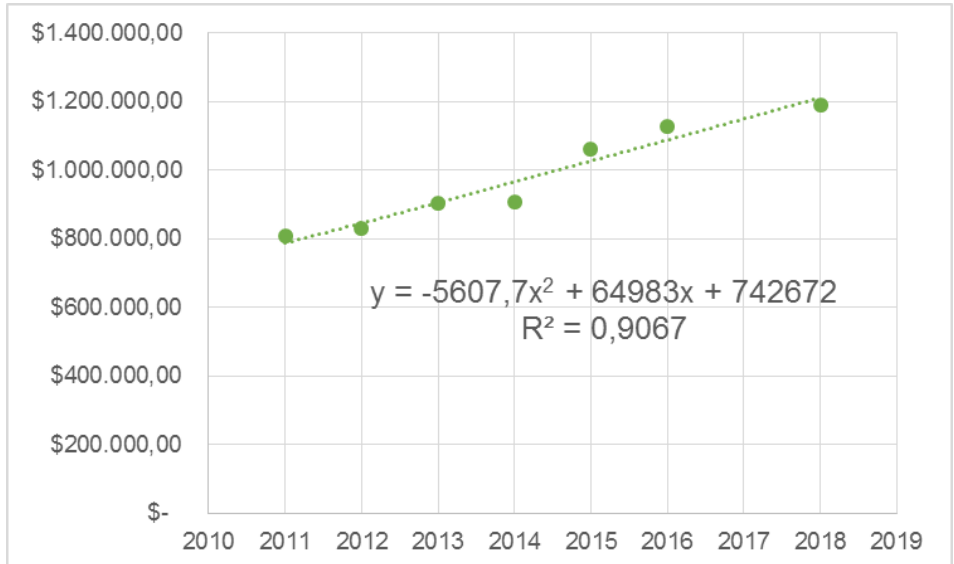

Figura 4: Proyección de ventas de la empresa 2 para los años 2015, 2016, 2018.

En la siguiente Tabla se puede evidenciar los montos que corresponderían a cada año mediante la proyección de tendencias polinómica.

Tabla 4: Proyección de ventas para la empresa 2 en los años 2015, 2016, 2018.

\begin{tabular}{ccc}
\hline Años & Ventas en USD. & Crecimiento / Decrecimiento ventas \% \\
\hline 2011 & $\$ 808.026,70$ & \\
2012 & $\$ 832.267,50$ & $3,00 \%$ \\
2013 & $\$ 905.090,91$ & $8,75 \%$ \\
2014 & $\$ 906.901,09$ & $0,20 \%$ \\
2015 & $\$ 1.061 .979,30$ & $17,10 \%$ \\
2016 & $\$ 1.126 .962,30$ & $6,12 \%$ \\
2018 & $\$ 1.191 .945,30$ & $5,77 \%$
\end{tabular}

Nota: Registros de ventas obtenidos de la Empresa 2 en estudio y su proyección utilizando proyección con tendencia polinómica.

Con respecto a lo que concierne a la Canasta Comercial Transfronteriza, se ha tomado en cuenta la información que cada una de las empresas proporcionó de cómo se ha hecho uso a cada uno de los cupos, no sin antes aclarar que las dos empresas tuvieron el cupo máximo que otorgaba la misma, en un monto de USD 67.928,00 (Ministerio de Comercio Exterior del Ecuador, 2015) en las dos veces que se aplicó en la provincia del Carchi. Con lo cual cada una de 
las empresas ocupó el cupo en su totalidad y se logró vender durante el periodo de vigencia de esta medida; es decir, entre el año 2015, 2016, 2017 (hasta el mes de mayo). La siguiente tabla muestra los montos de cómo se comportaron en estos tres años en las dos empresas.

Tabla 5: Desarrollo y comportamiento de la Canasta Comercial Transfronteriza en las dos empresas.

\begin{tabular}{ccccc}
\hline \multicolumn{5}{c}{ Empresa 1 } \\
\hline Año & Monto de importación & \% de ventas & Utilidad 28\% & Ingresos por ventas \\
2015 & $\$ 12.913,92$ & $19 \%$ & $\$ 3.615,90$ & $\$ 16.529,82$ \\
2016 & $\$ 31.265,28$ & $46 \%$ & $\$ 8.754,28$ & $\$ 40.019,56$ \\
$2017 *$ & $\$ 23.788,80$ & $35 \%$ & $\$ 6.660,86$ & $\$ 30.449,66$ \\
Total. & $\$ \mathbf{6 7 . 9 6 8 , 0 0}$ & $\mathbf{1 0 0 \%}$ & $\mathbf{\$ 1 9 . 0 3 1 , 0 4}$ & $\mathbf{\$ 8 6 . 9 9 9 , 0 4}$
\end{tabular}

Empresa 2

$\begin{array}{ccccc}\text { Año } & \text { Monto de importación } & \text { \% de ventas } & \text { Utilidad 32\% } & \text { Ingresos por ventas } \\ 2015 & \$ 8.835,84 & 13 \% & \$ 2.827,47 & \$ 11.663,31 \\ 2016 & \$ 37.382,40 & 55 \% & \$ 11.962,37 & \$ 49.344,77 \\ 2017 * & \$ 21.749,76 & 32 \% & \$ 6.959,92 & \$ 28.709,68 \\ \text { Total } & \mathbf{\$ 6 7 . 9 6 8 , 0 0} & \mathbf{1 0 0 \%} & \mathbf{\$ 2 1 . 7 4 9 , 7 6} & \mathbf{\$ 8 9 . 7 1 7 , 7 6}\end{array}$

Nota: Registros de montos y cupos de uso de las empresas en estudio de acuerdo al SRI. * Dato no oficial.

Según la información de la Tabla 6 se puede evidenciar que los montos y porcentajes de utilidad se pueden considerar como aceptables, tomando como referencia que en la empresa 1, la utilidad por cada producto importado bajo esta medida, tuvo un promedio de ganancia de $28 \%$; es decir, las ganancias fueron para esta empresa durante lo que duró la medida de USD 19.031,04, teniendo un ingreso en ventas para la empresa de USD 86.999,04.

Y para la empresa 2 el monto de ingresos por ventas refleja USD 89.717,76 para la empresa, dejando una utilidad de USD 21.749,76 durante los casi tres años que duró esta medida de ayuda por parte del gobierno.

Al momento del análisis de estos datos, y gracias a las proyecciones se puede hacer una comparativa entre los datos reales proporcionados por cada una de las empresas y las proyecciones que se realizaron con los montos que se esperaba obtener, siguiendo una línea de tendencia con respecto a las ventas que tenían en las empresas durante los años pasados.

En la siguiente tabla, se muestran los resultados de la Canasta Comercial Transfronteriza en comparación a las proyecciones de las empresas estudiadas.

Tabla 6: Resultados de la Canasta Comercial Transfronteriza en comparación a las proyecciones de las empresas estudiadas.

\section{Empresa 1}




\begin{tabular}{|c|c|c|c|c|c|}
\hline Año & $\begin{array}{c}\text { Ventas } \\
\text { obtenidas }\end{array}$ & $\begin{array}{c}\text { Ventas } \\
\text { esperadas }\end{array}$ & $\begin{array}{l}\text { Sesgo de } \\
\text { ingresos }\end{array}$ & $\begin{array}{c}\text { Canasta } \\
\text { Comercial }\end{array}$ & $\begin{array}{c}\% \text { de } \\
\text { compensación }\end{array}$ \\
\hline 2015 & $\$ 911.494,08$ & $\$ 1.060 .375,50$ & $-\$ 148.881,42$ & $\$ 16.529,82$ & $11,1 \%$ \\
\hline 2016 & $\$ 821.438,00$ & $\$ 1.089 .417,68$ & $-\$ 267.979,68$ & $\$ 40.019,56$ & $14,9 \%$ \\
\hline \multirow{3}{*}{$\begin{array}{c}2017 \\
*\end{array}$} & $\$ 672.405,00$ & $\$ 1.119 .038,62$ & $-\$ 446.633,62$ & $\$ 30.449,66$ & $6,8 \%$ \\
\hline & \multicolumn{2}{|l|}{ Total } & $-\$ 863.494,72$ & $\$ 86.999,04$ & $10,1 \%$ \\
\hline & & & Empresa 2 & & \\
\hline Año & $\begin{array}{c}\text { Ventas } \\
\text { obtenidas }\end{array}$ & $\begin{array}{c}\text { Ventas } \\
\text { esperadas }\end{array}$ & $\begin{array}{l}\text { Sesgo de } \\
\text { ingresos }\end{array}$ & $\begin{array}{c}\text { Canasta } \\
\text { Comercial }\end{array}$ & $\begin{array}{c}\% \text { de } \\
\text { compensación }\end{array}$ \\
\hline 2015 & $\$ 842.567,41$ & $\$ 1.061 .979,30$ & $-\$ 219.411,89$ & $\$ 11.663,31$ & $5,3 \%$ \\
\hline 2016 & $\$ 778.001,00$ & $\$ 1.126 .962,30$ & $-\$ 348.961,30$ & $\$ 49.344,77$ & $14,1 \%$ \\
\hline \multirow{2}{*}{$\begin{array}{c}2017 \\
*\end{array}$} & $\$ 668.290,00$ & $\$ 1.191 .945,30$ & $-\$ 523.655,30$ & $\$ 28.709,68$ & $5,5 \%$ \\
\hline & Total & & $-\$ 1.092 .028,49$ & $\$ 89.717,76$ & $8,2 \%$ \\
\hline
\end{tabular}

Nota: Registros de ventas obtenidos de las Empresas 1 y 2 en estudio en comparación con los resultados de la medida gubernamental. * Dato no oficial.

Es claro que los resultados presentados no guardan una relación adecuada con las ventas esperadas, pudiendo determinar que la mecánica de aplicación de la medida adoptada por el gobierno de turno en ese entonces, no cumplió con las expectativas de los empresarios investigados.

\section{Conclusiones}

Al finalizar el estudio, se ha podido llegar a constatar que la medida adoptada por el gobierno de turno denominada Canasta Comercial Transfronteriza, no tuvo el impacto esperado en las empresas investigadas, y extrapolando los resultados obtenidos y expresados anteriormente a las empresas consideradas grandes en la zona, también se puede expresar que la medida no cumplió con las expectativas del sector.

De acuerdo a la información levantada con los empresarios durante el presente trabajo investigativo, se pudo concluir que el mecanismo gubernamental implantado como medida para reactivación económica del sector, fue establecido de manera incorrecta pues permitía que todos los comerciantes del sector puedan importar cualquier tipo de productos, pese a no estar dentro de las actividades comerciales declaradas en su RUC, creando de esta manera competencia que los mismos empresarios lo catalogaron como desleal, afectando directamente a las empresas que operan bajo la ley en la importación de los productos que realmente estaban declarados en su RUC y que cotidianamente comercializan bienes dentro de su ámbito comercial.

Una medida que se implanta para reactivar zonas deprimidas debe ser bien estudiada, pero principalmente, previa a su aplicación, debe invitar a los beneficiarios potenciales para ser parte del equipo con el fin de encontrar posibles fallas en su propuesta y futura ejecución.

Las autoridades de gobierno tienen a su cargo una tarea muy importante al momento de plantear alternativas de reactivación económica para los diversos sectores, y es precisamente esto 
que obliga a que las decisiones sean tomadas de manera correcta y beneficiando a la gran mayoría, pero evitando a la vez posibles distorsiones o evasiones de la ley.

\section{Bibliografía}

Aparicio, G., \& Zorrilla, P. (2015). Distribución comercial en la era omnicanal. Madrid: Pirámide.

Colegio de Economistas. (2017). Comerciantes pedirán que se amplíe canasta comercial. Obtenido de http://colegiodeeconomistas.org.ec/noticias/mayo-22-2017/

COMEX. (2015). Ministerio de Comercio Exterior. Obtenido de http://www.comercioexterior.gob.ec/wp-content/uploads/2015/10/resolucion-0392015.pdf

Dapena Fernandez, J. L. (2015). Finanzas de la empresa. Toma de decisiones y subjetividad. Córdoba, Argentina: Asociación Cooperadora de la Facultad de Ciencias Económicas Universidad Nacional de Córdoba.

Diario El Telégrafo. (mayo de 2017). Obtenido de http://www.eltelegrafo.com.ec/noticias/economia/8/en-canasta-comercial-818comerciantes-del-norte-andino-movilizaron-mas-de-usd-4-millones

EKOS. (2016). Comerciantes de fronteras no están satisfechos con sus ventas. Obtenido de Revista EKOS: http://www.ekosnegocios.com/negocios/verArticuloContenido.aspx?idArt=8304

El Comercio. (2016). El Comercio. Obtenido de http://www.elcomercio.com/actualidad/canastacomercial-comerciantes-carchi-economia.html

Escuela de Tecnologías UDLA. (Octubre de 2016). Informativo de comercio Exterior №21. Quito, Ecuador.

ESP.

(2010).

Obtenido

de

http://halweb.uc3m.es/esp/Personal/personas/jmmarin/esp/GuiaSPSS/18reglin.pdf

Gemmel 1, N. (septiembre de 2001). Fiscal Policy in a Growth Framework.

Importsol. (2017). Importsol. Obtenido de https://www.solyavimport.com/tag/fodinfa/

Ministerio de Comercio Exterior del Ecuador. (2015). COMEX. Obtenido de http://www.comercioexterior.gob.ec/boletin-de-prensa-salvaguardia-por-balanza-depagos/

Porter, M. E. (2015). Estrategia competitiva. Madrid: Pirámide.

Render, B., Stair, R., \& Hanna, M. (2006). Métodos cuantitativos para los negocios. (Pearson, Ed.) México D.F, México. Obtenido de 
https://books.google.com.ec/books?id=oNuXccZkWfIC\&printsec=frontcover\&hl=es\#v= onepage $\& \mathrm{q} \& \mathrm{f}=$ false

Sapag, M. (2007). Proyectos de inversión, formulación y evaluación. (P. education, Ed.) México. 